\title{
Magnitude of opportunistic diseases and their predictors among adult people living with HIV enrolled in care: national level cross sectional study, Ethiopia
}

Teklu Weldegebreal $^{1}$, Ismael Ahmed ${ }^{1 *}$ (D), Abiyou Muhiye ${ }^{2}$, Shoandagne Belete ${ }^{2}$, Alemayehu Bekele ${ }^{2}$ and Mirgissa Kaba ${ }^{3}$

\begin{abstract}
Background: Opportunistic diseases cause morbidity and mortality among human immunodeficiency virus (HIV) infected persons. There is dearth of evidence on the magnitude and predictors of opportunistic diseases among PLHIV in Ethiopia. This study was conducted to determine the magnitude and predictors of opportunistic diseases among adults enrolled in the national HIV/AIDS care and treatment services and generate information for program planning and medicine quantification in the country.

Methods: A health facility-based cross-sectional study was conducted. Probability proportional to size and random sampling methods were employed to select health facilities and medical records of adult HIV-infected patients respectively. A total of 7826 medical records were reviewed from 60 health facilities nationwide. Socio-demographic and clinical data including diagnosis of opportunistic diseases were collected from the medical records. Period prevalence of opportunistic diseases over one year period was determined. Bivariate and multivariate logistic regression was used to measure associations between independent variables and the dependent variable, occurrence of opportunistic diseases.

Results: Of the total of 7826 study participants, 3748 (47.9\%) were from hospitals and 4078 were from health centers. The majority (61.8\%) were female. The median age was 32 years with interquartile range (IQR) of 27-40. The median duration of stay in HIV care was $56(\mathrm{IQR}=28-80)$ months; $7429(94.9 \%)$ were on antiretroviral treatment. A total of 1665 cases of opportunistic diseases were recorded with an overall prevalence estimated at 21.3\% (95\% confidence interval (CI): 20.36, 22.18\%). Skin diseases (4.1\%), diarrhea (4.1\%), bacterial pneumonia (3.6\%), recurrent upper respiratory tract infections (3.1\%) and tuberculosis (2.7\%) were the leading opportunistic diseases. Isoniazid preventive therapy coverage among eligible patients was 24.8\%. Persons with a CD4 count $<200$ cells $/ \mathrm{mm}^{3}$ [adjusted odds ratio (AOR) 1.80, 95\% Cl: $1.45,2.23]$; and who were bed ridden or ambulatory functional status [AOR $(95 \% \mathrm{Cl})=3.19(2.32,4.39)]$ were independent predictors of diagnosis of opportunistic diseases.
\end{abstract}

Conclusion: Opportunistic diseases were found to be pervasive among HIV infected adults in Ethiopia. Proactive identification and management, and prevention of opportunistic diseases should be strengthened especially among females, ambulatory or bed-ridden, and patients with low CD4 cell count.

Keywords: Opportunistic diseases, HIV, Ethiopia, Magnitude, Predictors, Cross sectional study

\footnotetext{
*Correspondence: xxa4@cdc.gov; ismaelahmed2003@gmail.com

${ }^{1}$ U.S. Centers for Disease Control and Prevention- Ethiopia, Addis Ababa,

Ethiopia

Full list of author information is available at the end of the article
}

(c) The Author(s). 2018 Open Access This article is distributed under the terms of the Creative Commons Attribution 4.0 International License (http://creativecommons.org/licenses/by/4.0/), which permits unrestricted use, distribution, and reproduction in any medium, provided you give appropriate credit to the original author(s) and the source, provide a link to the Creative Commons license, and indicate if changes were made. The Creative Commons Public Domain Dedication waiver (http://creativecommons.org/publicdomain/zero/1.0/) applies to the data made available in this article, unless otherwise stated. 


\section{Background}

Evidence on epidemiologic trend in low- and middle-income countries show a decline in the incidence and prevalence of opportunistic diseases following improvement in the quality of care services and the introduction of highly active antiretroviral treatment (ART) [1, 2]. Over the last decade, mortality among people living with human immunodeficiency virus (PLHIV) has decreased significantly [3]. In a study conducted at seven University-affiliated Hospitals in Ethiopia from January 2009-July 2013, mortality of 5.4/ 100 person-years of observation has been reported among adult PLHIV [4]. However, opportunistic diseases still remains the main cause of morbidity and mortality among PLHIV [5].

It is also evident that there is a disparity in the magnitude and types of opportunistic diseases between high- and low-income countries, and even between low-income countries themselves [6]. Thus, it is crucial for a country and a program to determine the types and magnitudes of opportunistic diseases affecting PLHIV in order to deliver impactful interventions and effectively manage resources.

Ethiopia has accomplished much in terms of HIV service expansion and uptake, which impacted on prevention and control of human immunodeficiency virus (HIV) and acquired immunodeficiency syndrome (AIDS) over the last decade. Ethiopian Public Health Institute (EPHI) estimated a total of 724,400 PLHIV with adult HIV prevalence of $1.2 \%$ for the year 2014 [7]. The Federal Ministry of Health (FMoH) 2014/2015 Annual Performance Report shows 375,811 PLHIV were enrolled and actively retained in the national ART program [8].

However, despite the progress, little evidence exists regarding the magnitude, and distribution of opportunistic diseases among PLHIV in Ethiopia. Available studies [9-12] and their estimates so far have been based on data from limited health facilities, small number of patients and varying time periods; thus limiting their generalizability and utility for national use. As a result, planning treatment and prevention interventions, developing and quantifying an evidencebased prioritized list of medicine and diagnostics has been a challenge. Therefore, this national level study was conducted to generate data on the type, magnitude and predictors of opportunistic diseases among adult PLHIV who were enrolled into the national HIV/AIDS care and treatment program. Information from this study will be used to facilitate program planning, and inform resource planning, specifically the quantification and distribution of medicines needed for management of opportunistic diseases in Ethiopia.

\section{Methods}

\section{Study setting}

Chronic HIV/AIDS care and treatment services in Ethiopia are provided at hospital and health center settings throughout the country. As of Dec. 2013, the FMoH adopted the 2013 World Health Organization (WHO) recommendation and moved ART eligibility criteria from $\leq 350$ to $\leq 500 \mathrm{CD} 4$ cell count irrespective of the WHO clinical stage. The chronic HIV/AIDS care service includes screening and management of opportunistic diseases. This study was conducted at national level by selecting representative public health facilities providing chronic HIV/AIDS care services irrespective of their geographic location using probability proportional to size (PPS) methodology.

\section{Study design}

A health facility-based, cross-sectional study was carried out between September 2013 and August 2014. Medical records of HIV-infected adults who received pre-ART and/or ART care services in public health facilities in Ethiopia were reviewed.

\section{Study participants}

The study participants were adult (age $\geq 15$ years old) HIV-infected who were enrolled in care and received pre-ART or ART care and treatment services at least once between September 2013 and August 2014 in the selected public health facilities.

\section{Sample size and sampling procedure}

The sample size was determined by employing the following formula and assumptions:

$$
n=Z_{\frac{a}{2}}{ }^{2}\left(\frac{P(1-P)}{d^{2}}\right) x D
$$

$\mathrm{P}=$ Prevalence of clinically diagnosed Cryptococcus infection among PLHIV in Addis Ababa public hospitals was documented to be $3.7 \%$ [13].

$\mathrm{d}=$ margin of error between sample and population (0.006)

$\mathrm{Z}_{\alpha / 2}=$ critical value at $95 \%$ confidence interval (1.96)

$\mathrm{D}=$ design effect for a cluster sampling of hospitals and health centers (1.5)

Accordingly, sample size was estimated at 6267 . During piloting of data collection procedures at two health facilities in Addis Ababa, we noted poor documentation of medical records. Therefore, an additional 30\% allowance was made to compensate for poor documentation of medical records making the total sample size for the assessment to be 8147 .

The number of health facilities and medical records needed to be reviewed from each sample facility was determined by considering the resources required to 
conduct the study and also calculating the limits of precision of the estimates. As a result, we determined that if 60-80 facilities are selected and higher than 75 medical records are reviewed from each facility, the precision of the estimate of these values will be \pm 0.006 or higher. Therefore, we decided to sample 60 health facilities and review 136 medical records from each of them.

A two-stage, stratified sampling method was employed to sample the required health facilities and medical records. To select health facilities we used the President's Emergency Plan for AIDS Relief (PEPFAR)-Ethiopia country office September 2013 report which listed 832 health facilities (710 health centers and 122 hospitals) with their PLHIV load from all regions of Ethiopia [14]. First, we prepared the sampling frame by stratifying health facilities into health center and hospital. Then, PPS sampling of facilities [15] was used to select health facilities from each stratum, independently; 31 health centers and 29 hospitals were selected using the PPS methodology. Medical records from each selected health facilities were selected using simple random sampling (SRS). Sample medical records were selected from electronic list of medical records (sampling frame) of pre-ART and ART patients in each of selected health facilities. Random numbers were generated using Excel sheet to select the required number of medical records from each facility. Schematics of sampling procedure is depicted in Fig. 1.

\section{Data collection tools and procedures}

Prior to commencement of data collection, qualified data collectors and supervisors were recruited and trained on the contents of data abstraction tools, procedures and ethical standards; and data collection tools and procedures were field tested at two health facilities (1 hospital and 1 health center).

Upon arrival at the selected health facilities, the data collecting team met with ART program focal person and data management personnel and briefed them the study objectives and data collection processes. Data was collected from patients' medical records by using a structured, paper-based, data abstraction tool. The tool captured data on socio-demographic variables including sex, age, education and marital status; clinical measurement including recent CD4 count, nutritional status, functional status and WHO clinical staging [16]; ART status and adherence to medications; diagnosis and treatment for opportunistic diseases; provision of isoniazid preventive therapy (IPT) and cotrimoxazole prophylaxis; and last documented follow-up status.

\section{Definitions of terms}

Nutritional status: was determined by computing body mass index (BMI) as $\mathrm{kg} / \mathrm{m}^{2}$. WHO classification was used to categorize participants: as severe thinness $\left(\right.$ BMI $\left.<16.00 \mathrm{~kg} / \mathrm{m}^{2}\right)$, moderate thinness (BMI 16.00$16.99 \mathrm{~kg} / \mathrm{m}^{2}$ ), mild thinness (BMI $17.00-18.49 \mathrm{~kg} / \mathrm{m}^{2}$ ), normal (BMI $18.50-24.99 \mathrm{~kg} / \mathrm{m}^{2}$ ), overweight (25.00$\left.29.99 \mathrm{~kg} / \mathrm{m}^{2}\right)$ and obese $\left(\mathrm{BMI} \geq 30.00 \mathrm{~kg} / \mathrm{m}^{2}\right)$. Patients with severe, moderate or mild thinness were categorized as undermatron.

Functional status: was defined in line with the definition on the national HIV care follow-up form: working functional status - able to perform usual work in and out of the house; ambulatory - able to perform activities of daily living but not able to work; and bedridden - not able to perform daily routine activities.

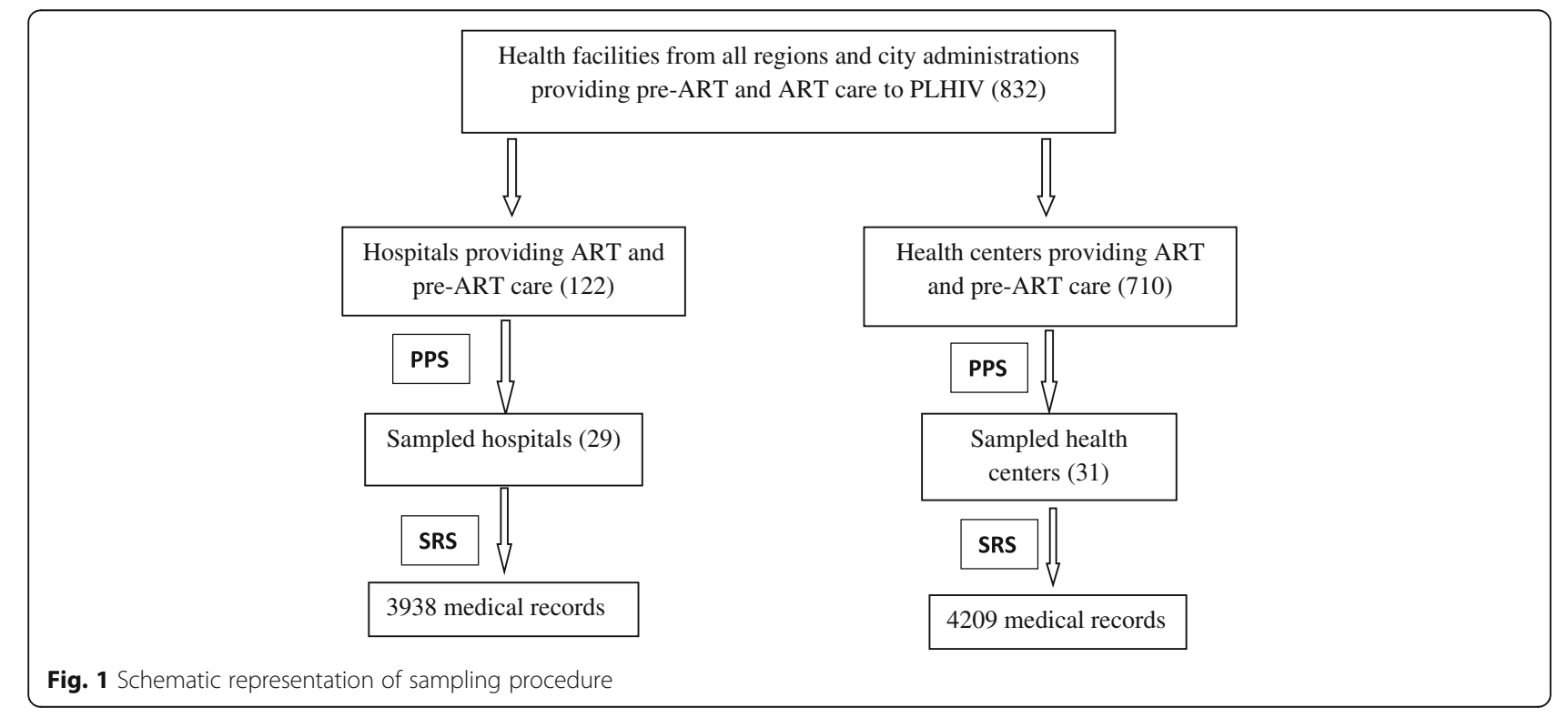


Adherence: was defined in line with the definition on the national HIV care follow-up form, adherence to ART was classified as good, fair or poor. Good adherence is defined as taking $>95 \%$ of prescribed doses, fair adherence as taking $85-94 \%$ of prescribed doses, and poor adherence as taking $<85 \%$ of prescribed doses. In this study, we defined good adherence to ART during the study period if the participant had constant good adherence status; fair adherence if the participant had at least one documented fair adherence status; and poor adherence if the participant had at least one documented poor adherence status throughout his/her follow-up during study period. Pre-ART and ART patients have follow-up visit to HIV clinic every six and three months on average, respectively.

Follow-up status: in line with the national HIV care follow-up form, a patient was considered lost to follow-up if the patient do not have a follow-up visit within 30 days after the latest clinic appointment date. If a patient moved to another health facility with confirmed written documentation of transfer out, follow-up was defined as transferred out. Death was ascertained only if documented in one of the reviewed medical records.

Tuberculosis (TB) symptom screening at last follow-up visit was used to categorize study participants into symptom screen result positive or negative. Occurrence of symptoms of cough, fever, night sweat and weight loss were the symptoms used for screening. Participants were categorized as symptom screen positive if they reported at least one of the above four symptoms during screening.

Opportunistic diseases were defined in accordance with the lists of opportunistic diseases indicated on the Ethiopian National Guideline for Comprehensive HIV Prevention, Care and Treatment [17]. The study only collected information on opportunistic diseases that were diagnosed between September 2013 and August 2014.

\section{Data management and quality assurance}

Supervisors ensured adherence to standards of data collection at facility level by supervising data abstraction and checking for data quality in accordance with a standard data collection manual. Data quality issues identified onsite were addressed immediately. Data was checked for completeness and consistency, and double entered by two data entry clerks to ensure data quality.

\section{Data analysis}

Data were entered into Epi Info software 3.5.1 to clean and ensure consistency of the data set. Clean data were exported to and analyzed using SPSS version 21. Descriptive statistics were used to analyze study participants socio-demographic and clinical parameters; the mean and standard deviation were calculated for normally distributed continuous variables and frequency (\%) was used for categorical variables. Median and interquartile range was calculated for continuous variables with skewed distribution.

Opportunistic diseases documented on medical records of patients were used to analyze the types and frequencies of opportunistic diseases. The period prevalence of opportunistic diseases was determined as the proportion of adult PLHIV who developed one or more opportunistic diseases during the study period.

Bivariate statistics were employed to determine distribution of the study subjects by independent variable and to measure crude association between independent variables (sex, age group, recent CD4 count, taking prophylactic medications for opportunistic diseases, nutritional status, care status, follow-up status, and functional status) and the occurrence of opportunistic diseases. Odds ratios (OR) with 95\% CI were used as a measure of association, and $p$-value of $<0.05$ was considered statistically significant. Multivariate analysis was used to determine the independent effects of selected variables on opportunistic disease diagnosis controlling for the effects of others using complete case analysis. However, nutritional status measurement which was significantly affected by missing data (32\%) was not included in the analysis. Independent predictor variables with a $\mathrm{p}$-value $<0.05$ were used for the final model and their association was calculated as adjusted odds ratio (AOR) with their 95\% CI. Site-level clustering was not accounted in the analysis.

\section{Results}

The socio-demographic characteristics of the study participants are presented in Table 1 . The study reviewed medical records of 7826 adult PLHIV who had at least one follow-up visit at public health facilities in Ethiopia over the 12 months period, September 2013 to August 2014. The medical record retrieval rate was $96.1 \%$ of the planned records for review. Of the total participants, 3748 (47.9\%) attended care and treatment services at hospitals and $4078(52.1 \%)$ at health centers. The median age of study participants was $32(\mathrm{IQR}=27-40)$ years. Females were relatively younger than males, median age of 30 and 36 years respectively. Nearly two third of participants (61.8\%) were female. Among the 7109 with recorded marital status, more than half of participants 3612 (50.8\%) were married and 2432 (34.2\%) were either separated/divorced or widowed. Among the 7070 with recorded educational status, more than a third (34.9\%) had no education, while $31.4 \%$ had secondary or more education.

\section{Clinical characteristics of study participants}

The clinical characteristics of the study participants are presented in Table 2. The median time in HIV care was 
Table 1 Socio-demographic characteristics of adult PLHIV in Ethiopia, September 2013 to August 2014

\begin{tabular}{|c|c|}
\hline Characteristics & Number (\%) \\
\hline \multicolumn{2}{|c|}{ Health facilities where patients enrolled into care } \\
\hline Hospital & $3748(47.9)$ \\
\hline Health Center & $4078(52.1)$ \\
\hline Total & $7826(100.0)$ \\
\hline \multicolumn{2}{|l|}{ Sex } \\
\hline Male & $2989(38.2)$ \\
\hline Female & $4837(61.8)$ \\
\hline Total & $7826(100.0)$ \\
\hline \multicolumn{2}{|l|}{ Age group } \\
\hline $15-24$ & $957(12.2)$ \\
\hline $25-34$ & $3365(43.0)$ \\
\hline $35-44$ & $2339(29.9)$ \\
\hline $45-54$ & $868(11.1)$ \\
\hline$>55$ & $297(3.8)$ \\
\hline Total & $7826(100.0)$ \\
\hline \multicolumn{2}{|l|}{ Marital status } \\
\hline Never married & $1065(15.0)$ \\
\hline Married & $3612(50.8)$ \\
\hline Divorced/separated & $1604(22.6)$ \\
\hline Widow/widower & $828(11.6)$ \\
\hline Total & $7109(100.0)$ \\
\hline \multicolumn{2}{|l|}{ Education } \\
\hline No education & $2469(34.9)$ \\
\hline Primary & 2384 (33.7) \\
\hline Secondary & $1662(23.5)$ \\
\hline Tertiary & $555(7.9)$ \\
\hline Total & 7070 (100.0) \\
\hline
\end{tabular}

$56(\mathrm{IQR}=28-80)$ months. Of the 7429 (94.9\%) study participants on ART, 7346 (98.9\%) were on first-line ART regimen and $83(1.1 \%)$ were on second-line. The majority, $5689(88.2 \%)$, of those participants who were on ART had consistent good adherence to their treatment throughout the follow-up period. Among the 7735 with recorded WHO's clinical staging, 855 (11.1\%) of clients were found to be at advanced clinical stages (stage III or IV). Of the 7730 with recorded functional status, 251 (3.2\%) were either bed-ridden or ambulatory. Among the 5337 with recorded nutritional status, 1192 (22.3\%) participants were undernourished. The median CD4 count for the total study population was 407 (IQR $=261-572)$ cells $/ \mathrm{mm}^{3}$. Of the $6187(79.1 \%)$ participants whose CD4 count was done and documented, 951 (15.4\%) had CD4 count less than 200 cells $/ \mathrm{mm}^{3}$.

TB symptom screening coverage at last visit was documented for 7612 (97.3\%) participants; 7184 (94.4\%) of
Table 2 Clinical characteristic of adult PLHIV in public health facilities of Ethiopia, September 2013 to August 2014

\begin{tabular}{|c|c|}
\hline Characteristics & Number (Percentage) \\
\hline \multicolumn{2}{|l|}{ Care status } \\
\hline On pre-ART care & $397(5.1)$ \\
\hline On ART & $7429(94.9)$ \\
\hline Total & $7826(100.0)$ \\
\hline \multicolumn{2}{|l|}{ WHO clinical staging } \\
\hline Stage $|/| \mid$ & $6880(88.9)$ \\
\hline Stage III/IV & $855(11.1)$ \\
\hline Total & $7735(100.0)$ \\
\hline \multicolumn{2}{|l|}{ Functional status } \\
\hline Working & 7479 (96.8) \\
\hline Ambulatory/bed ridden & $251(3.2)$ \\
\hline Total & $7730(100.0)$ \\
\hline \multicolumn{2}{|l|}{ Nutritional status } \\
\hline Severe/moderate thinness & $421(7.9)$ \\
\hline Mild thinness & $771(14.4)$ \\
\hline Normal & $3430(64.3)$ \\
\hline Overweight/obese & $715(13.4)$ \\
\hline Total & $5337(100.0)$ \\
\hline \multicolumn{2}{|l|}{ CD4 count (cells/mm³) } \\
\hline$<200$ & $951(15.4)$ \\
\hline $200-349$ & $1553(25.1)$ \\
\hline $350-499$ & $1556(25.1)$ \\
\hline$\geq 500$ & $2127(34.4)$ \\
\hline Total & $6187(100.0)$ \\
\hline \multicolumn{2}{|l|}{ TB symptoms screening result } \\
\hline Positive & $428(5.6)$ \\
\hline Negative & $7184(94.4)$ \\
\hline Total & $7612(100.0)$ \\
\hline \multicolumn{2}{|l|}{ Isoniazid prophylaxis } \\
\hline Yes & $1703(24.8)$ \\
\hline No & $5170(75.2)$ \\
\hline Total & $6873(100.0)$ \\
\hline \multicolumn{2}{|l|}{ Adherence to ART } \\
\hline Good & $5689(88.2)$ \\
\hline Poor/fair & $764(11.8)$ \\
\hline Total & $6453(100.0)$ \\
\hline \multicolumn{2}{|l|}{ Cotrimoxazole prophylaxis } \\
\hline Yes & $5135(97.1)$ \\
\hline No & $154(2.9)$ \\
\hline Total & $5289(100.0)$ \\
\hline Median duration of stay in HIV care (months) & $56(\mathrm{IQR}=28-80)$ \\
\hline Median CD4 count (cells/ mm³) & $407(\mathrm{IQR}=261-572)$ \\
\hline
\end{tabular}


screened participants had no TB symptoms. Of the 7184 with no TB symptoms, 6873 (95.6\%) were eligible for IPT. However, IPT was provided for only 1703 (24.8\%) of those eligible.

\section{Follow-up status of study participants in HIV care}

Last follow-up status was documented for 7794 (99.6\%) of the participants. As shown in Fig. 2, 6944 (89\%) of the participants were in active follow-up, $316(4.1 \%)$ had been transferred out to other health facilities, 446 (5.7\%) were lost to follow-up and 88 (1.1\%) had died.

\section{Types and magnitudes of opportunistic diseases}

A total of 1665 cases of opportunistic diseases were recorded for the study period, with a prevalence of $21.3 \%$ (95\% CI: 20.36, 22.18\%). We found 264 (3.4\%) medical records which had documented chief complaints that may or may not associate with opportunistic diseases, but with no documented diagnosis. These medical records were treated as records with no opportunistic diseases diagnosis.

Of those diagnosed with opportunistic diseases 917 (55.1\%) sought care at a hospital and $748(44.9 \%)$ at a health centers. The proportion of participants with opportunistic diseases during study period categorized by year of their enrollment into the care service is shown in Fig. 3, the proportion of opportunistic disease among patients recently enrolled into HIV care was higher, estimated at $39 \%$.

Types of opportunistic diseases diagnosed per patient ranged from one to six disease types: most $(80.1 \%)$ had one type, $16.4 \%$ had two types and the remaining $2.3 \%$ had three and more types. The number of clinic visits per patient for diagnosis and management of opportunistic diseases ranged from one to eleven visits.

As shown in Table 3, skin diseases (4.1\%), diarrhea (4.1\%), bacterial pneumonia (3.6\%), recurrent upper



respiratory tract infections $(3.1 \%)$ and $\mathrm{TB}(2.7 \%)$ were the leading opportunistic diseases. Severe forms of opportunistic infections were diagnosed more at hospitals than health centers: bacterial infections including bacterial meningitis and sepsis [OR $(95 \% \mathrm{CI}): 1.95(1.23,3.08)]$, bacterial pneumonia [OR (95\% CI): $2.55(1.98,3.28)$ ], and toxoplasma encephalitis [OR (95\% CI): 9.25 (2.14, 40.00)]. Of a total of 206 TB cases, 160 (77.7\%) were pulmonary $\mathrm{TB}, 26(12.8 \%)$ were extra-pulmonary $\mathrm{TB}$, and $20(9.7 \%)$ were disseminated TB cases.

As shown in Fig. 4, bacterial skin diseases (30.3\%) such as cellulitis, impetigo and carbuncles were the most common skin diseases followed by minor mucocutaneous infections (28.7\%).

\section{Predictors of opportunistic diseases}

As shown in Table 4, in the multivariate logistic regression analysis participants with CD4 count $<200 / \mathrm{mm}^{3}$, sex, and ambulatory or bed-ridden functional status were independently associated with opportunistic diseases. Those with a CD4 count $<200$ cells $/ \mathrm{mm}^{3}$ were $80 \%$ more likely to have opportunistic disease compared to those with CD4 count $\geq 500$ cells $/ \mathrm{mm}^{3}$, [AOR (95\% CI): $1.80(1.45,2.23)]$; males were $14 \%$ less likely to have opportunistic diseases compared to females, [AOR (95\% CI): 0.86 (0.74, 0.99)]; and PLHIV with either bed ridden and ambulatory functional status were 3.2 time more likely to be diagnosed with opportunistic diseases compared to those with working functional status, [AOR (95\% CI): $3.19(2.32,4.39)]$.

\section{Discussion}

This cross-sectional study determined the common types of opportunistic diseases prevailing among PLHIV and their magnitude. Predictors for the occurrence of opportunistic diseases have also been outlined.

This study elucidated the proportion of opportunistic diseases affecting adult PLHIV in Ethiopia was 21.3\% (95\% CI- 20.36, 22.18\%). This finding is comparable with $19.7 \%$ prevalence reported among adult PLHIV attending care services at Gondar University Hospital [9] and 22.4\% prevalence reported from South East Nigeria [18]. However, it is lower than the prevalence reported from Hiwot Fana Hospital, 48.0\% [10] and Debre Markos Hospital, 42.8\% [11]. The observed difference between the current study and those reported higher prevalence could be due to differences in diagnostic capacity, study period or methods used to retrieve and analyze the data. The study from Hiwot Fana Hospital was not period specific while the study from Debre Markos Hospital reported on prevalence of opportunistic diseases during five to seven year on HAART follow-up and excluded diseases which patients initially presented with. 

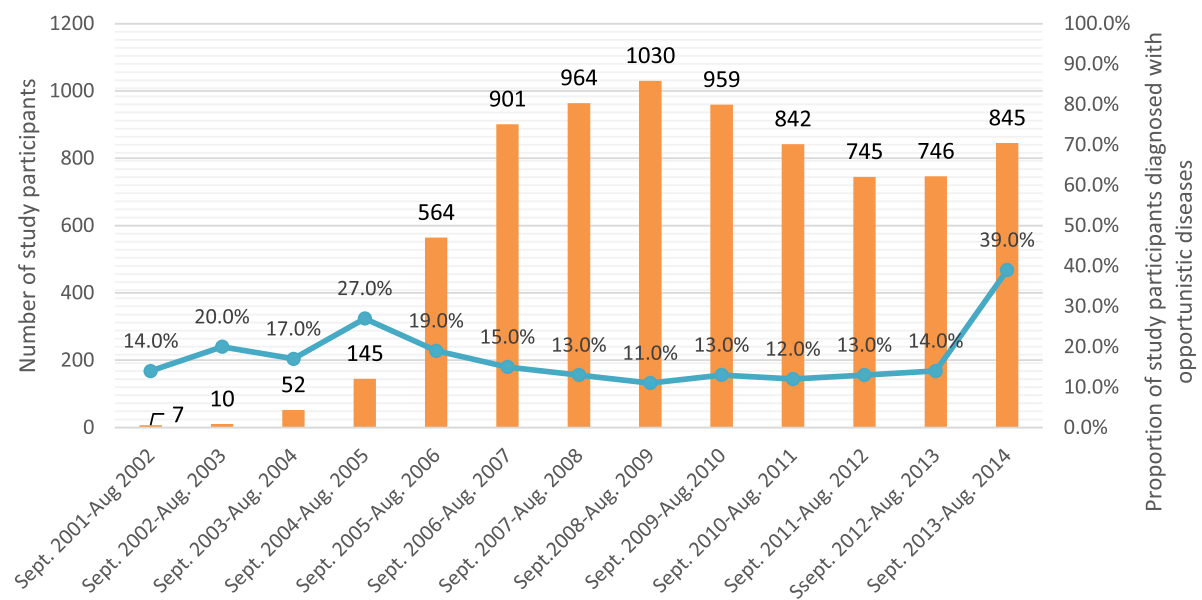

Year of enrolment in to HIV care

study participant $\quad$ - $\quad$ \% with opportunistic diseases

Fig. 3 Proportion of adult PLHIV with OI by year of enrollment, September 2013 to August 2014

The current study also showed that proportion of opportunistic diseases among the newly enrolled adult PLHIV was higher than those who stayed in HIV care for a year or more. This could be because opportunistic diseases might have prompted patients to seek medical care at their initial visit or have manifested following enrollment in to care. The proportion (39.0\%) observed in the current study was lower than the report from Felege Hiwot Hospital [12] which reported prevalence of $88.9 \%$ among ART-naïve adult PLHIV. The observed difference between the two studies could be due to changes in
PLHIV care seeking behavior, access to ART services or the time period in which studies were conducted: in 2008 PLHIV were presenting to health facilities with advanced immune-compromise [mean $(95 \% \mathrm{CI})$ for CD4 count: 153 cells $\left./ \mathrm{mm}^{3}(139,167)\right]$ and ART was offered for those with CD4 count less than 200 cells $/ \mathrm{mm}^{3}$ as opposed to the current study. The current study also showed that the prevalence of opportunistic diseases among those stayed in care for more than seven years is relatively higher than those who were in care for more than a year but less than seven years; possibility of

Table 3 Types and proportions of opportunistic diseases diagnosed among study participant during one year follow-up period, September 2013 to August 2014

\begin{tabular}{lllll}
\hline Opportunistic Disease & $\begin{array}{l}\text { Frequency } \\
\text { (prevalence) }\end{array}$ & $\begin{array}{l}\text { Frequency at hospital } \\
\text { (prevalence) }\end{array}$ & $\begin{array}{l}\text { Frequency at health } \\
\text { Center (prevalence) }\end{array}$ & $\begin{array}{l}\text { OR, at hospitals compared } \\
\text { to health centers (95\% Cl) }\end{array}$ \\
\hline Skin diseases & $318(4.1 \%)$ & $169(4.5 \%)$ & $149(3.7 \%)$ & $1.13(0.90,1.43)$ \\
Diarrhea & $317(4.1 \%)$ & $126(3.4 \%)$ & $191(4.5 \%)$ & $0.66(0.52,0.83)$ \\
Bacterial pneumonia & $281(3.6 \%)$ & $197(5.3 \%)$ & $84(2.1 \%)$ & $2.35(1.80,3.05)$ \\
Recurrent upper respiratory tract infections & $242(3.1 \%)$ & $113(3.0 \%)$ & $129(3.2 \%)$ & $0.88(0.68,1.14)$ \\
Tuberculosis & $206(2.7 \%)$ & $98(2.6 \%)$ & $108(2.6 \%)$ & $0.91(0.69,1.20)$ \\
Oro-pharyngeal candidiasis & $123(1.6 \%)$ & $82(2.2 \%)$ & $41(1.0 \%)$ & $2.00(1.37,2.92)$ \\
Severe bacterial infection & $78(1.0 \%)$ & $50(1.3 \%)$ & $28(0.7 \%)$ & $1.79(1.12,2.85)$ \\
Perianal problems & $31(0.4 \%)$ & $23(0.6 \%)$ & $8(0.2 \%)$ & $2.88(1.28,6.44)$ \\
Toxoplasma Gondi encephalitis & $19(0.2 \%)$ & $17(0.5 \%)$ & $1(0.0 \%)$ & $8.50(1.96,36.84)$ \\
Pneumocystis pneumonia & $13(0.2 \%)$ & $12(0.3 \%)$ & $1(0.0 \%)$ & $12.00(1.56,92.37)$ \\
Herpes simplex & $12(0.2 \%)$ & $11(0.3 \%)$ & $1(0.0 \%)$ & $11.00(1.42,85.28)$ \\
Cryptoccoccal meningitis & $8(0.1 \%)$ & $7(0.2 \%)$ & $1(0.0 \%)$ & $7.00(0.86,56.95)$ \\
Lymphoma & $2(0.0 \%)$ & $1(0.0 \%)$ & $4(0.1 \%)$ & $1.00(0.06,16.00)$ \\
Other Ols & $15(0.2 \%)$ & $11(0.3 \%)$ & $748(18.3 \%)$ & $2.75(0.87,8.65)$ \\
Total & $1665(21.3 \%)$ & $917(24.5 \%)$ & $1.23(1.09,1.37)$
\end{tabular}




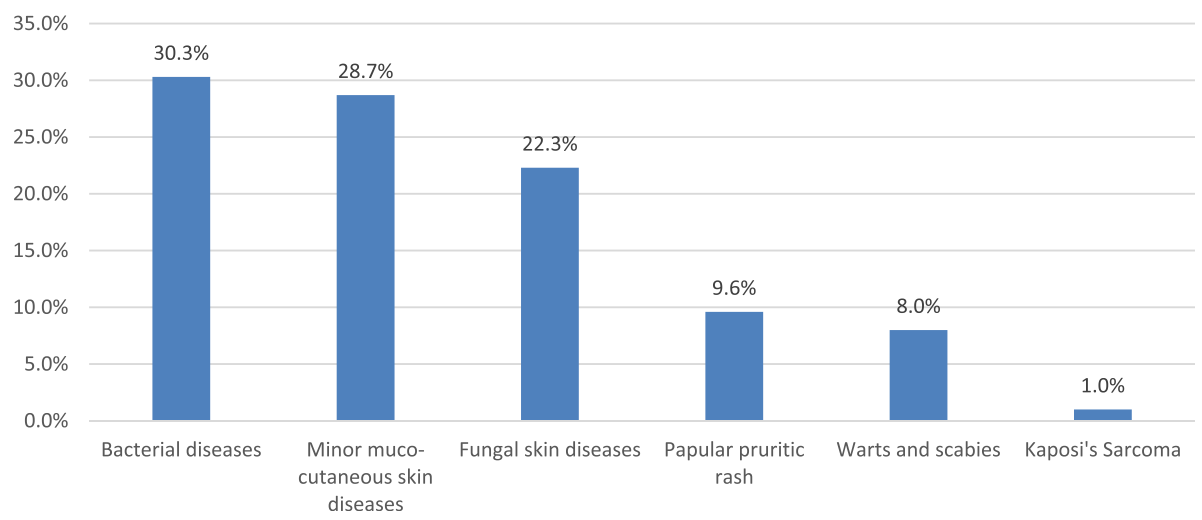

Fig. 4 Distribution of skin diseases among adult PLHIV in Ethiopia, September, 2013 to August, 2014

Table 4 Predictors of opportunistic diseases among adult PLHIV, September 2013 to August 2014

\begin{tabular}{|c|c|c|c|c|}
\hline Characteristics & COR $(95 \% \mathrm{Cl})$ & $P$-value & AOR $(95 \% \mathrm{Cl})$ & $P$-value \\
\hline \multicolumn{5}{|l|}{$\operatorname{Sex}(n=7826)$} \\
\hline Male & $0.917(0.810,1.038)$ & 0.169 & $0.86(0.74,0.99)$ & $0.047^{*}$ \\
\hline Female & 1.00 & & & \\
\hline \multicolumn{5}{|l|}{ Age group $(n=7826)$} \\
\hline $15-24$ & 1.00 & & & \\
\hline $25-34$ & $0.81(0.67,0.98)$ & $0.029^{*}$ & $0.83(0.61,1.03)$ & 0.092 \\
\hline $35-44$ & $0.82(0.68,1.00)$ & 0.052 & $0.89(0.71,1.12)$ & 0.304 \\
\hline $45-54$ & $0.82(0.64,0.1 .04)$ & 0.104 & $0.80(0.61,1.07)$ & 0.134 \\
\hline$>55$ & $0.97(0.70,1.36)$ & 0.879 & $0.93(0.63,1.38)$ & 0.719 \\
\hline \multicolumn{5}{|l|}{ Recent CD4 $(n=6187)$} \\
\hline$<200$ & $2.18(1.81,2.61)$ & $0.001^{*}$ & $1.80(1.45,2.23)$ & $0.001^{*}$ \\
\hline $200-349$ & $1.02(0.85,1.22)$ & 0.851 & $0.99(0.81,1.21)$ & 0.939 \\
\hline $350-499$ & $0.95(0.79,1.14)$ & 0.556 & $0.94(0.78,1.14)$ & 0.529 \\
\hline$\geq 500$ & 1.00 & & & \\
\hline \multicolumn{5}{|l|}{ Care status $(n=7826)$} \\
\hline In pre-ART care & $1.72(1.36, .2 .18)$ & $0.001^{*}$ & $1.33(0.95,1.85)$ & 0.093 \\
\hline In ART & 1.00 & & & \\
\hline $\begin{array}{l}\text { Taking any prophylactic medication } \\
\text { for opportunistic infection }\end{array}$ & 1.00 & & & \\
\hline Yes & $0.84(0.74,0.96)$ & 0.008 & $0.92(0.77,1.08)$ & 0.296 \\
\hline \multicolumn{5}{|l|}{ No } \\
\hline Follow-up status $(n=7826)$ & 1.00 & & & \\
\hline On active follow-up & $1.11(0.83,1.50)$ & 0.480 & $0.82(0.56,1.19)$ & 0.292 \\
\hline Transferred-out & $1.54(1.22,1.95)$ & $0.001^{*}$ & $1.29(0.93,1.79)$ & 0.125 \\
\hline Lost to follow-up & $5.66(3.71,8.64)$ & $0.001^{*}$ & $4.16(2.26,7.66)$ & $0.001^{*}$ \\
\hline \multicolumn{5}{|l|}{ Died } \\
\hline \multicolumn{5}{|l|}{ Recent functional status ( $n=7730$ ) } \\
\hline Working & 1.00 & & & \\
\hline Ambulatory or bed-ridden & $4.75(3.68,6.13)$ & 0.001 & $3.19(2.32,4.39)$ & $0.001^{*}$ \\
\hline
\end{tabular}

*Significant at $p$-value $<0.05$ 
increased risk of ART failure in those group should be considered.

Regarding the types and magnitude of opportunistic diseases, the six major leading opportunistic diseases identified in this study were skin infections (4.1\%), bacterial pneumonia (3.6\%), recurrent upper respiratory tract infection (3.1\%), tuberculosis (2.7\%) and oropharyngeal candidiasis $(1.6 \%)$. The pattern is different from the previous studies conducted in Northwest and Eastern part of Ethiopia [9-12]. This could be due to methodological differences with respect to operational definitions and also differences between study settings where all of the previous studies were single hospitalbased studies. Our evaluation also showed differences in magnitude of those diseases between health facility types: bacterial pneumonia, skin diseases and diarrhea, consecutively, were the leading prevalent opportunistic disease among PLHIV attending at hospitals while diarrhea, skin diseases and recurrent upper tract infections were common among attendants at health centers. Moreover, it has elucidated that severe types of opportunistic diseases were less diagnosed in health center settings compared to hospitals. The differences between hospitals and health centers could be related with diagnosing capacity and quality of services, wherein hospitals have more doctors and greater diagnostic infrastructure compared to health centers, or it may be that sicker PLHIV tend to seek care more often at hospitals than health centers.

We found that skin diseases were the leading causes of morbidity in adult PLHIV in Ethiopia. Bacterial and fungal skin infections contributed $52.5 \%$ of the observed skin diseases. This finding was consistent with the study conducted in Brighton, department of HIV medicine [19] who reported infections as a leading causes of skin diseases among PLHIV followed by dermatosis, pruritus and malignancy; though the prevalence of skin diseases in their study was higher, 91.4\%.

The proportion PLHIV diagnosed with of TB (2.7\%) in this study is lower than reports from other similar studies which reported prevalence of 7.7\% [18], 9.7\% [9] and $18.2 \%$ [10]. Similar with a study conducted at Gondar University Hospital, [9] the majority of patients diagnosed with $\mathrm{TB}$ in this study had pulmonary form of the disease.

The current study found that despite high TB symptom screening coverage (97.3\%), IPT service was provided only for $24.8 \%$ of eligible PLHIV; this finding is comparable to $19.6 \%$ IPT coverage reported from hospitals in Tigray Regional State of Ethiopia [20]. Drug availability and distribution, health care providers' perception on importance of the service, and acceptability of the service among the PLHIV have been implicated as challenges to the program [21]. This low service coverage is a timely alarm to closely examine barriers to the IPT service and intensify the TB/HIV service package.

The study showed that low current CD4 count $<200$ cells $/ \mathrm{mm}^{3}$ was strongly associated with increased morbidity with opportunistic diseases, similar to studies from Ethiopia and other sub-Saharan Africa [9, 10, 18].

This study also showed that sex was independently associated with occurrence of opportunistic diseases. Female PLHIV were more likely to have opportunistic diseases compared to their male counterparts. Given female sex is disproportionately affected by HIV and majority $(61.8 \%)$ of PLHIV in the care system are female [8] implications of this finding is paramount.

Lost to follow-up from the care services was found to be strongly associated with increases in the probability of diagnosis with opportunistic diseases. This finding is consistent with our observations in the health facilities, and studies reporting opportunistic diseases being among risk factors for lost to follow-up and deaths [4, 22-24].

Similar with a hospital-based study conducted at Hiwot Fana Hospital, Eastern part of Ethiopia [10], this study reports opportunistic diseases are significantly associated with PLHIV being bed ridden or ambulatory. This could be explained by lack or limited mobility of patients who may begin to lose interest in eating because they are not getting enough stimulation on a regular basis and unable to care for themselves.

PLHIV in pre-ART care had an increased association with opportunistic diseases as compared to those on ART, concordant with the study conducted at Gondar University Hospital [9]. In addition, the lost to follow-up rate was higher among PLHIV in pre-ART care $(43.1 \%)$ compared to those on ART (3.4\%); this finding is comparable to $38.4 \%$ lost to follow-up rate reported by a study that addressed the pre-ART care status in Ethiopia [22].

This national level cross sectional study was the first to our knowledge to establish national level information pertaining to opportunistic diseases among adult PLHIV enrolled in care. It has used a sufficient sample size with wider geographical coverage and health facility type representation to ensure generalizability; indicated the national level prevalence of opportunistic diseases, revealed the types, magnitude and predictors of opportunistic diseases as well as the coverage of critical care and support services; and estimated prevalence in a way that helps inform the procurement and distribution of medications for health facilities. However, due to the fact that this study was conducted using available medical records, retrospectively, it is subjected to limitations that emanate from incomplete recording of PLHIV clinical information on their records, and diagnostic capacity of health facilities involved in the study. Improvement in 
patients' medical information documentations is one of the major issues the country health system need to work on. In addition to this, the OD identified in this study may not show the true picture of the common opportunistic infections prevalent among PLHIV in Ethiopia. This could be due to the fact that almost all of the study population were taking ART in addition to other OD preventing prophylaxis treatments.

This study has not assessed association of viral load status of participants with opportunistic diseases for the test was not performed routinely. Initiating and/or scaling up of routine viral load testing service at all ART service provision points is crucial to determine the association of viral load test result with opportunistic diseases and rate of treatment failure especially among PLHIV who have been on ART for long period of time.

\section{Conclusion}

This study demonstrated that opportunistic diseases were pervasive among adult PLHIV enrolled in care in Ethiopia and distribution of opportunistic diseases was different from reports of previous studies conducted in the Eastern and Northwest part of Ethiopia. Future national level endeavors to prioritize quantification, procurement and distribution of medicines for the prevention and treatment of opportunistic diseases should take findings of this study into consideration.

CD4 count $<200 / \mathrm{mm}^{3}$, female sex, and ambulatory or bed-ridden functional status were independent predictors of opportunistic diseases. Opportunistic disease screening and prevention services should prioritize those PLHIV groups. Strategies and care models that emphasize early identification new PLHIV and accelerated implementation of test-and-treat approach including differentiated care models should be strengthened.

Only quarter of eligible PLHIV were on IPT. Importance of improving IPT service coverage in the country cannot be over emphasized. Strategies to mitigate challenges pertaining to IPT program and improve its service coverage should also be explored and implemented.

\section{Abbreviations}

AIDS: Acquired Immunodeficiency Syndrome; ART: Antiretroviral Therapy; BMI: Body Mass Index; CDC: Centers for Disease Control and Prevention; EDHS: Ethiopia Demographic and Health Survey; EPHA: Ethiopian Public Health Association; EPHI: Ethiopian Public Health Institute; FMoH: Federal Ministry of Health; HIV: Human Immunodeficiency Virus; IPT: Isoniazid Preventive Therapy; OI: Opportunistic Infection; PEPFAR: President's Emergency Plan for AIDS Relief; PLHIV: People Living with HIV; TB: Tuberculosis; WHO: World Health Organization

\section{Acknowledgements}

This study has been supported by the PEPFAR through the US CDC under the terms of Cooperative Agreement with EPHA number GH001039-03.

The authors are grateful to Mr. Habtamu Woldeamanuel and Mr. Bekele Belayhun for their contribution during data collection and management. The heads and ART clinic focal persons of health facilities, included in this study are acknowledged for their unreserved effort in making the data accessible to the study team. The authors also extend our heartfelt gratitude to the data collectors and supervisors who took part in the field activities.

\section{Funding}

The study was funded by US Centers for Disease Control and Prevention (CDC).

Availability of data and materials

The datasets used and/or analyzed during the current study are available from EPHA on reasonable request.

\section{Authors' contributions}

TW had primary responsibility in the process of conceptualization and research design, data analysis and interpretation, and manuscript drafting and revision. IA participated in the revision of study protocol and design, development of data collection instruments, interpretation of results, and manuscript drafting and revision. AM participated in the development of study protocol and design, coordination of the overall study, development of data collection instruments, data collection and analysis, and drafting of the study report. SB coordinated the overall study, participated in conceptualization and research design, participated in the preparation and revision of the manuscript. $A B$ participated in the write up of the study finding and critical review of the other sections. MK was the principal investigator of the study and had a responsibility in the process of conceptualization and research design, data analysis and interpretation. All authors read and approved the final manuscript.

\section{Authors' information}

TW: MD, MSc; Senior Public Health Specialist at CDC Ethiopia. He has expertise in HIV/AIDS treatment, care and support. IA: MPH; Public Health Specialist at CDC Ethiopia. He has expertise in HIV/AIDS treatment, care and support. AM: MD, MPH; Research Coordinator at EPHA. SB: BSC, MPH: Research Coordinator at EPHA. He is a registered and licensed chief expert Public Health Professional. AB: MPH, PhD; Projects and Grants Management Department Director at EPHA. MK: MA, PhD; Asst Prof. of Public Health at the School of Public Health, Addis Ababa University.

\section{Ethical approval and consent to participate}

The study protocol was reviewed by EPHA and U.S Centers for Disease Control and Prevention (CDC) and granted ethical clearance by both institutions. The commencement of the study was facilitated by official letter written by the $\mathrm{FMoH}$ and EPHA to the regional health authorities and health facilities. Data were collected from medical records of patients with no personal identifiers; and therefore no consent was sought from individuals. Medical records were accessed with permission from the heads of the respective health authorities at regional and health facility level. Only data collectors and supervisors accessed medical records and data collection took place in a secure place within the facility to avoid a breach of confidentiality. Care was taken not to disrupt routine clinic activities during data collection.

\section{Consent for publication}

Not applicable.

\section{Competing interests}

The authors declare that they have no competing interests.

\section{Publisher's Note}

Springer Nature remains neutral with regard to jurisdictional claims in published maps and institutional affiliations.

\section{Author details}

${ }^{1}$ U.S. Centers for Disease Control and Prevention- Ethiopia, Addis Ababa, Ethiopia. ${ }^{2}$ Ethiopian Public Health Association, Addis Ababa, Ethiopia. ${ }^{3}$ School of Public Health, Addis Ababa University, Addis Ababa, Ethiopia.

Received: 20 October 2017 Accepted: 21 June 2018

Published online: 03 July 2018

\section{References}

1. Low A, Gavriilidis G, Larke N, B-Lajoie M-R, Drouin O, Stover J, et al. Incidence of opportunistic infections and the impact of antiretroviral therapy among HIV-infected adults in low- and middle-income countries: a systematic review and meta-analysis. Clin Infect Dis. 2016;62(12):1595-603. 
2. Coelho L, Cardoso SW, Amancio RT, Moreira Rl, Campos DP, Veloso VG, et al. Trends in AIDS-defining opportunistic illnesses incidence over 25 years in Rio de Janeiro. Brazil PLoS ONE. 2014;9(6):e98666.

3. Reniers G, Slaymaker E, Nakiyingi-Miiro J, Nyamukapa C, Crampin AC, Herbst $K$, et al. Mortality trends in the era of antiretroviral therapy: evidence from the network for Analysing longitudinal population based HIV/AIDS data on Africa (ALPHA). AIDS. 2014;28(Suppl 4):S533-42.

4. Daniel F, Teklu W, Alula MT, Melake D, Saro A, Nega B, et al. Predictors of Survival among Adult Ethiopian Patients in the National ART Program at Seven University Teaching Hospitals: A Prospective Cohort Study. Ethiop J Health Sci. 2017;27(si1):63-71. https://doi.org/10.4314/ejhs.v27i1.7S.

5. Simmons RD, Ciancio BC, Kall MM, Rice BD, Delpech VC. Ten-year mortality trends among persons diagnosed with HIV infection in England and Wales in the era of antiretroviral therapy: AIDS remains a silent killer. HIV Medicine. 2013;14(10):596-604

6. Iroezindu MO. Disparities in the magnitude of human immunodeficiency virus-related opportunistic infections between high and low/middle-income countries: is highly active antiretroviral therapy changing the trend? Annals of Medical and Health Sciences Research. 2016;6(1):4-18.

7. Ethiopian Public Health Institute: HIV Related Estimates and Projections for Ethiopia- 2015. Addis Ababa, Ethiopia.

8. Federal Ministry of Health E. Health Sector Development Programme IV: Annual Performance Report. 2015.

9. Damtie D, Yismaw G, Woldeyohannes D, Anagaw B. Common opportunistic infections and their CD4 cell correlates among HIV-infected patients attending at antiretroviral therapy clinic of Gondar University hospital. Northwest Ethiopia BMC Research Notes. 2013;6(1):1-7.

10. Mitiku H, Weldegebreal F, Teklemariam Z. Magnitude of opportunistic infections and associated factors in HIV-infected adults on antiretroviral therapy in eastern Ethiopia. HIV/AIDS (Auckland, NZ). 2015;7:137-44.

11. Moges NA, Kassa GM. Prevalence of opportunistic infections and associated factors among HIV positive patients taking anti-retroviral therapy in Debre Markos referral hospital, Northwest Ethiopia. J AIDS Clin Res. 2014;5:301. https://doi.org/10.4172/2155-6113.1000301.

12. Bayeh A, Fisseha W, Tsehaye T, Atnaf A, Mohammed Y. ART-naive HIV-infected patients at Feleg-Hiwot referral hospital northwest, Ethiopia. Ethiopian J of Health Dev. 2010;24:3-8.

13. Beshah G. Study of prevalence of opportunistic infections among HIV/AIDS patients in Addis Ababa public hospitals [Thesis for partial fulfilment of the requirments for the degree of Masters of Public Health]2011.

14. Office PEC. List of health facilities providing ART and pre ART services with their client load. 2013

15. Bennett $\mathrm{S}$, Woods T, Liyanage WM, Smith DL. A simplified general method for cluster-sample surveys of health in developing countries. World Health Stat Q. 1991;44(3):98-106.

16. $\mathrm{WHO}$ case definitions of HIV for surveillance and revised clinical staging and immunological classification of HIV-related disease in adults and children [Internet]. [cited 8, Sept 2016]. Available from: http://www.who.int/hiv/pub/ guidelines/hivstaging/en/.

17. Federal Ministry of Health E. National Guideline for comprehensive HIV prevention, Care and treatment. 2014

18. Iroezindu MO, Ofondu EO, Hausler H, Wyk BV. Prevalence and risk factors for opportunistic infections in HIV patients receiving antiretroviral therapy in a resource-limited setting in Nigeria. J AIDS Clinic Res. 2013;S3:002. https://doi.org/10.4172/2155-6113.S3-002.

19. Uthayakumar S, Nandwani R, Drinkwater T, Nayagam AT, Darley CR. The prevalence of skin disease in HIV infection and its relationship to the degree of immunosuppression. Br J Dermatol. 1997;137(4):595-8.

20. Teklay G, Teklu T, Legesse B, Tedla K, Klinkenberg E. Barriers in the implementation of isoniazid preventive therapy for people living with HIV in northern Ethiopia: a mixed quantitative and qualitative study. BMC Public Health. 2016;16(1):1-9.

21. Getahun H, Granich R, Sculier D, Gunneberg C, Blanc L, Nunn P, et al. Implementation of isoniazid preventive therapy for people living with HIV worldwide: barriers and solutions. AIDS. 2010;24(Suppl 5):S57-65.

22. Loha DSWTAAYBBMAMTSDHE. Pre ART status in Ethiopia: performance and challenges. Hawassa University School of Public and Environ Health, 2014.
23. Tanuma J, Lee KH, Haneuse S, Matsumoto S, Nguyen DT, Nguyen DTH, et al. Incidence of AIDS-defining opportunistic infections and mortality during antiretroviral therapy in a cohort of adult HIV-infected individuals in Hanoi, 2007-2014. PLoS One. 2016;11(3):e0150781.

24. Biadgilign S, Reda AA, Digaffe T. Predictors of mortality among HIV infected patients taking antiretroviral treatment in Ethiopia: a retrospective cohort study. AIDS Res Ther. 2012;9(1):1-7.

\section{Ready to submit your research? Choose BMC and benefit from:}

- fast, convenient online submission

- thorough peer review by experienced researchers in your field

- rapid publication on acceptance

- support for research data, including large and complex data types

- gold Open Access which fosters wider collaboration and increased citations

- maximum visibility for your research: over $100 \mathrm{M}$ website views per year

At BMC, research is always in progress.

Learn more biomedcentral.com/submissions 